\title{
A Novel 20G Wide-Band Synthesis Methodology for CMOS Spiral Inductors using Neural Network and Genetic Algorithm
}

\author{
Haiyang Shen Wenjun Zhang Tao Liu \\ CAD Department, Institute of Microelectronics, Tsinghua University, Beijing 100084, China
}

\begin{abstract}
We develop a novel synthesis way to effectively generate CMOS spiral inductor's layout parameters using artificial neural network and genetic algorithm. An accurate neural network model for CMOS spiral inductors is firstly developed based on measured results from TSMC $0.13 \mathrm{um} \mathrm{MM} / \mathrm{RF}$ process with the frequency range of 1-20 GHz. The neural network model is further integrated in the synthesis simulator kits called SPUNK. An innovative synthesis technique is then applied in which genetic algorithm based optimization is adopted. Our methodology promises to provide greater accuracy than previous results in the frequency range while able to minimize the time cost for spiral inductor design.
\end{abstract}

Keywords: CMOS spiral inductor, Neural network, Genetic algorithm, Layout parameter optimization.

\section{Introduction}

In recent years, there has been a great development in the CMOS RF integrated circuit due to the large demand of communication industry such as wireless communication. Compared with other integrated circuit processes, CMOS process has the advantage of high density and low power [1]. But passive components with large area and high power consumption make the design of CMOS RF integrated circuit a great challenge, especially the lack of inductors with high $\mathrm{Q}$ value. The most used on-chip inductors are spiral inductors for their low cost and ease of process [2]. As the operation frequency increases, various physical mechanisms such as skin effect, proximity effect and electric-magnetic penetration into substrate make spiral inductors difficulty to be well modeled and designed [3].

Recently, a lot of research has been done on the modeling and synthesis of spiral inductor and there are mainly two ways of inductors modeling maturely developed: Numerical Simulation and Empirical models. Artificial Neural Network methodology is a newly developed way which can calculate the results fast and accurately in a wide frequency range once the network has been well trained and becomes more and more popular in the modeling of RF passive devices [4] [5].

Synthesis, the converse of modeling, is of great importance, too. The aim of synthesis is to work out the process parameters and layout geometrical parameters according to the design parameters such as value of inductance, value of $\mathrm{Q}$ and so on. The general methodology of inductor synthesis is such a loop that gets the design parameters by making use of the simulator and then optimizes the process parameters and layout geometrical parameters to get the most satisfactory results by various optimization ways. According to the kinds of simulator, the way of synthesis can also be classified into three kinds: synthesis based on Numerical Simulation [5], synthesis based on empirical models [3] [7] [8] and synthesis based on Neural Network [1]. Among the three ways, the methodology based on empirical models is mostly used because there is a lot of mature research results focused on empirical models, not only analytical expressions but also equivalent circuits. The methodology based on Neural Network, as a novel way for fast and accurate synthesis, has attracted more and more attention and is used in synthesis of other devices and circuits [9], [10]. Although the advantages and disadvantages of these synthesis ways are largely dependent on their simulators under this loop optimization methodology, the choice of better optimization way is of great importance, too. Traditional analytic optimization way such as Levenberg-Marquardt method [11], artificial intelligent algorithm such as Genetic Algorithm [12], [13] and Particle Swarm Optimization (PSO) [14] and some other multi-objective optimization techniques are put into use [8].

In this paper, a novel Layout-level Synthesis Methodology for Integrated Spiral Inductors is proposed. It uses the artificial neural network as the simulator and the synthesis adopts Genetic Algorithm as the optimization way. We carry out experiments 
based on the octagon differential spiral inductors of TSMC 0.13um MM/RF process. On account of the rapid and accurate neural network simulator and excellent global optimization capacity of genetic algorithm, this methodology is able to synthesis layout parameters fast and accurately in frequency up to $20 \mathrm{GHz}$, including the turns of spiral, $\mathrm{n}$; the width of metal line, w; the spacing between the metal lines, s; the inner radius, $r$, which is of great help to the designers of RF Integrated Circuits.

\section{Neural Network Simulator SPUNK}

The block diagram of the function of the neural network simulator SPUNK (Simulation Program Using Neural Network) for spiral inductors is illustrated in Fig. 1. It is a modeling tool of RF passive devices based on both $\mathrm{C}++$ and Matlab programming language. In RF circuit design, the electrical characteristics of passive components are generally presented using $\mathrm{S}$ parameters for the ease of measurement. Therefore, $\mathrm{S}$ parameters are used as our model output. After training, SPUNK can calculate the S parameters of the two-port network of a spiral inductor according to the input of the layout geometrical parameters including the turns of spiral, n; the width of metal line, w; the spacing between the metal lines, $s$; the inner radius, $r$ and the operating frequency. Besides, since S12 and S21 are equal for a passive two-port reciprocal network like spiral inductor, S12 and S21 is theoretical the same and therefore only S 12 is included in the model output.

SPUNK adopts the Levenberg-Marquardt (LM) method is utilized as the training algorithm. Although not shown here, our experiments indicate that the LM method is a significantly faster and preferable algorithm to other methods such as the conjugategradient (CG), quasi-Newton, and back-propagation momentum algorithms. In addition, to ensure the validity and speed of simulation under higher frequency than the Self-Resonance Frequency (SRF) of the inductor, SPUNK adopts unique physics-based sampling technique. In this procedure, the $\mathrm{S}$ parameters for each inductor are simulated from low operation frequency to high frequency. The Inductance $\mathrm{L}$ of each inductor is accordingly calculated. Once the calculated inductance becomes negative, this frequency point will be excluded from training data and the higher operation frequency will no longer be simulated for this inductor.

Figure.1: The function of SPUNK.

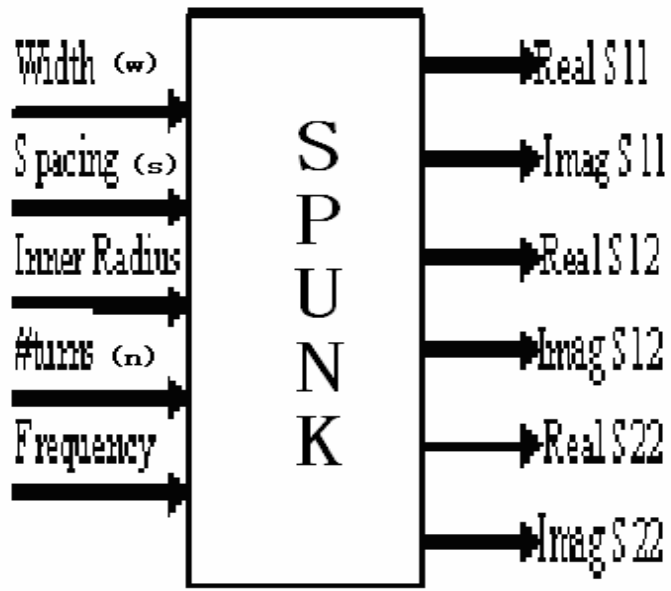

After S parameters are given by the neural network simulator, the electrical design parameters of inductors can be calculated according to the following expressions gained from the equivalent network.

Firstly, convert the $\mathrm{S}$ parameter matrix to $\mathrm{Y}$ parameter matrix:

$$
Y=\frac{1}{Z_{0}}[I-S][I+S]^{-1}
$$

Secondly, calculate the electrical design parameters of the inductor using the $\mathrm{Y}$ parameter matrix:

$$
\begin{gathered}
L_{11}=\frac{1}{\omega} \operatorname{Im}\left(1 / Y_{11}\right) \\
L_{22}=\frac{1}{\omega} \operatorname{Im}\left(1 / Y_{22}\right) \\
Q_{22}=\frac{\operatorname{Im}\left(\frac{1 / Y_{22}}{\operatorname{Re}\left(\frac{1}{Y_{22}}\right)}=-\frac{\operatorname{Im}\left(Y_{22}\right)}{\operatorname{Re}\left(Y_{22}\right)}\right.}{Q_{11}=\frac{\operatorname{Im}\left(1 / Y_{11}\right)}{\operatorname{Re}\left(\frac{1}{Y_{11}}\right)}=-\frac{\operatorname{Im}\left(Y_{11}\right)}{\operatorname{Re}\left(Y_{11}\right)}}
\end{gathered}
$$

and Q11 are the value of inductance and value of quality factor seen from port1; L22 and Q22 are the value of inductance and value of quality factor seen from port2. In ideal cases (the shape and process is symmetric enough), L11 are equal to L22 and Q11 are equal to Q22, too. So in the following calculation, we choose L11 and Q11 as the inductance and quality factor of the inductor.

\section{Optimization using Genetic Algorithms}


Genetic algorithm is a guided stochastic search technique based on mechanics of evolution and natural Selection [15]. They operate through creation of generations of "population" of strings, evaluation and selection of "most fit" strings, and generic manipulation to create a new population. The strings are formed by encoding of each variable and through the operation of crossover and mutation all the possible combinations of the solution space can be searched and evaluated, so Genetic algorithms has good global optimization capability. Besides, Genetic algorithm is intrinsically parallel, so the optimization process is faster than other analytical numerical optimization ways.

Based on our synthesis process, every step of genetic algorithm is deliberately designed as follows:

1. Population creation. A reasonable population size can make the optimization process search the solution space more thoroughly, thereby reducing the chance that the algorithm will return a local minimum that is not a global minimum, as well as make the algorithm to run more quickly. In our optimization process, we create population with uniform function and set the population size of generation to be 30 .

2. Objective and Fitness function. Our synthesis objective is to match the target $\mathrm{L}$ as accurate as possible and make the quality factor $\mathrm{Q}$ of the inductor maximum under the constraints of layout area. This is in nature a multi-objective optimization problem. By introducing reasonable power index, we can transform the multi-objective problem into a single-objective one with constraint. Formulated mathematically is:

$$
\min F=P_{1} \times\left|L-L_{\text {spec }}\right|+P_{2} / Q_{\text {spec }}
$$

$$
\text { const.Area } \leq \text { Areaspec }
$$

In the function, Lspec, Qspec and Areaspec are the synthesis targets. P1 and P2 are power index for $\mathrm{L}$ and $\mathrm{Q}$ respectively, the precise value of which will be set during the process according to different optimization targets. Generally speaking, we will set lower value for $\mathrm{P} 2$, for example $\mathrm{P} 1=0.1$ and $\mathrm{P} 2=1$ because design of RF IC needs inductors of quality factor while is less sensitive to the variation of the inductance L. In addition, the scaling of fitness function should be taken into consideration in the set of power index, for the too large range of fitness value caused by inappropriate value of power index will result in the early-convergence of genetic optimization.

3. Fitness scaling. As mentioned above, appropriate range of fitness value is very important for the convergence of the optimization process. Fitness scaling processes the raw fitness value of individuals into an appropriate range. The way of fitness scaling we adopt is Rank methodology.

4. Selection operation. We choose the mostly used Roulette Wheel strategy as our selection function which is described by:

$$
P \text { select }-i=F_{i} / \sum_{i} F_{i}
$$

In the above formulation, $P_{\text {select_ } i}$ is the final possibility of selection while $F_{i}$ is the fitness value of one sample after scaling and the sum of $F_{i}$ is the total fitness value of the present generation.

5. Crossover and reproduction operation. Crossover operation is an important way to enlarge population diversity and find the global optimization in the solution space by exchanging the corresponding part of two parents' generic code. Scattered crossover, single-point crossover, two-point crossover are frequently used for Crossover operation, in which twopoint crossover can We choose two-point as our crossover strategy because it can promote the search in the solution space more thoroughly and prevent the early convergence to the local minimum and we set the crossover possibility as 0.8 . In additional, every generation we will retain 2 best individuals (elite) to next generation

4. Mutation operation. We use an adaptive strategy called adaptive feasible for mutation, i.e., to set a large mutation possibility value in the beginning of optimization and set it smaller to converge to the final solution.

\section{Methodology and Flow of Synthesis}

The aim of inductor synthesis is to get the layout-level geometrical parameters according to the target electrical design parameters such as $\mathrm{L}, \mathrm{Q}$ and so on. The four layout-level parameters of CMOS spiral inductors are: $\mathrm{n}$, the turns of spiral; $\mathrm{w}$, the width of metal line; $s$, the spacing between the metal lines; $r$, the inner radius. Based on the artificial neural network and genetic algorithm methodology, the methodology and flow of synthesis we adopt is shown in figure.2 [16]:

1. Input the electrical parameters of inductor to be optimized.

The electrical parameters of inductor to be optimized is the inductor value $L$ and the quality value $\mathrm{Q}$ under the constraint of the inductor area.

2. Optimization process. 
Optimization process is an iterative process between the Genetic Optimizer and the Neural Network SPUNK. Because the turn $\mathrm{n}$ is discrete, we set $\mathrm{n}$ as an appropriate value before hand. Therefore, in the real optimization process, only w, s, r are being optimized in the Genetic Optimizer. The electrical parameters L, Q of every generations of individual are extracted in the Neural Network SPUNK. Then the

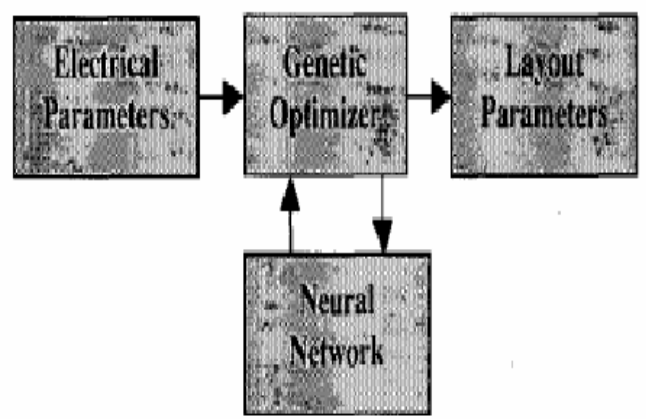

Figure 2: NEURO-genetic optimization PROCESS [16].

electrical parameters will be processed in the Genetic Optimizer. After generations of optimization, the optimum layout parameters will be output finally.

\section{Experiments and results}

Our synthesis experiments are based on the octagon differential spiral inductors of TSMC 0.13um MM/RF process and the geometrical shape of an example inductor is seen in Figure.3 [12].

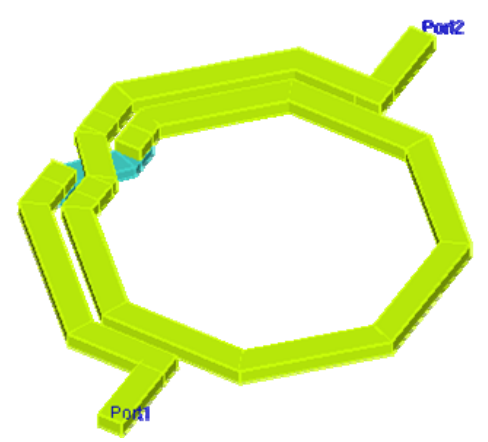

Figure.3. An example of octagon differential spiral inductors (1.5 turns)

According to our synthesis flow, at first, about 5,000 design samples are chosen from the design space and the range and the step of the layout parameters of the inductor is listed in the Table I .

Then, the correspondent L, Q, area of each design sample is calculated using SPUNK. Compare these values of inductance with the goal inductance and select the best results in Q and area.

\begin{tabular}{|c|c|c|}
\hline Parameter & Ranges & Step \\
\hline $\mathrm{N}$ & $1.5-5.5$ & 1 \\
\hline $\mathrm{W}(\mathrm{um})$ & $2-10$ & 1 \\
\hline S (um) & $2-6$ & 1 \\
\hline R (um) & $50-70$ & 1 \\
\hline
\end{tabular}

Table 1:The range and the step of the layout parameters.

All the following experiments are carried out on a computer with 2.67GHz Pentium CPU and 448MB memory.

Example1: An inductor of $2.2 \mathrm{nH}$ with min $\mathrm{Q}$ of 10 operating at the frequency of $2.45 \mathrm{GHz}$ is synthesized. 14 design samples meet such requirements and their correspondent parameters are listed in Table II.

\begin{tabular}{l|l|l|l|l|l|l}
\hline \hline Experiment & $\mathrm{Q}$ & $\mathrm{N}$ & $\mathrm{W}$ & $\mathrm{S}$ & $\mathrm{R}$ & Area \\
\hline 1 & 10.35 & 2.5 & 7 & 2 & 61 & 2625 \\
\hline 2 & 10.18 & 2.5 & 7 & 6 & 62 & 2684 \\
\hline 3 & 10.68 & 2.5 & 8 & 5 & 63 & 2991 \\
\hline 4 & 11.20 & 2.5 & 9 & 3 & 64 & 3314 \\
\hline 5 & 11.01 & 2.5 & 9 & 4 & 64 & 3314 \\
\hline 6 & 11.47 & 2.5 & 10 & 2 & 65 & 3653 \\
\hline 7 & 11.33 & 2.5 & 10 & 3 & 65 & 3653 \\
\hline \hline
\end{tabular}

Table 2: Synthesis results of one inductor.

Among these results, no.6 achieves max $Q$ with area of $3653 \mathrm{um}^{2}$. So we choose $\mathrm{n}=2.5, \mathrm{w}=10, \mathrm{~s}=2$, $\mathrm{r}=65$ as the optimization result. The whole synthesis process costs $7.2 \mathrm{~s}$.

Some other synthesis examples are carried out with different $\mathrm{Q}$ and different operating frequency. The results are seen in Table III.

\begin{tabular}{|l|l|l|l|l|l|l|l|l|}
\hline Experiment & $\mathrm{L}$ & $\mathrm{Q}$ & $\mathrm{f}$ & $\mathrm{N}$ & $\mathrm{W}$ & $\mathrm{S}$ & $\mathrm{R}$ & Time(s) \\
\hline 1 & 10 & 9.94 & 2.45 & 5.5 & 8 & 3 & 52 & 7.5 \\
\hline 2 & 2 & 10.28 & 5 & 2.5 & 7 & 3 & 62 & 6.9 \\
\hline 3 & 10 & 4.52 & 5 & 4.5 & 6 & 2 & 68 & 7.0 \\
\hline 4 & 2 & 6.81 & 10 & 2.5 & 9 & 3 & 59 & 6.9 \\
\hline 5 & 10 & 0.87 & 10 & 4.5 & 9 & 6 & 52 & 7.7 \\
\hline 6 & 2 & 4.72 & 15 & 2.5 & 5 & 5 & 54 & 6.9 \\
\hline 7 & 10 & 1.19 & 15 & 3.5 & 6 & 2 & 53 & 7.0 \\
\hline 8 & 2 & 4.20 & 17 & 2.5 & 5 & 5 & 51 & 7.0 \\
\hline 9 & 4 & 3.1 & 17 & 2.5 & 5 & 5 & 51 & 7.0 \\
\hline 10 & 1 & 9.38 & 20 & 1.5 & 9 & 2 & 67 & 7.0 \\
\hline
\end{tabular}

Table 3: Synthesis results of inductors.

The results above show the rule that $\mathrm{L}$ and $\mathrm{Q}$ are contradictive: when $\mathrm{L}$ is large, $\mathrm{Q}$ must be low and vice versa. All of the synthesis is finished in about $7 \mathrm{~s}$, which indicate our synthesis methodology is a fast and effectively way.

\section{Conclusion}


A novel wide-band layout-level synthesis methodology based on coupled Artificial Neural Network and generic algorithm is developed in this paper. Due to the high speed and high accuracy of Artificial Neural Network simulator SPUNK and the global optimization ability of genetic algorithm, the synthesis of layout-level spiral inductors becomes simple and fast with high accuracy. A lot of experiments are done to prove the advantages of this methodology. This novel synthesis approach is suitable for being employed in the design and layout optimization of inductors of RF circuits.

\section{References}

[1] S. Mukherjee, B. Mutnury and S. Dalmia, Layout-Level Synthesis of RF Inductors and Filters in LCP Substrates for Wi-Fi Applications, IEEE TRANSACTIONS ON MICROWAVE THEORY AND TECHNIQUES, 53(6), JUNE 2005.

[2] Y. Cao, R.A. Groves, and Xuejue Huang, Frequency-Independent Equivalent-Circuit Model for On-Chip Spiral Inductors, IEEE JOURNAL OF SOLID-STATE CIRCUITS, 38(3), MARCH 2003.

[3] N. A. Talwalkar, C.P. Yue, and S.S. Wong, Analysis and Synthesis of On-Chip Spiral Inductors , IEEE TRANSACTIONS ON ELECTRON DEVICES, 52(2), FEBRUARY 2005.

[4] T. Liu, Research of Artificial Neural Network Based Modeling Approach for RF Components, Dissertation Submitted to Tsinghua University in partial fulfillment of the requirement for the degree of Master of Engineering, 2006.

[5] T. Liu, W.J. Zhang, and Z.P. Yu, Modeling of Spiral Inductors Using Artificial Neural Network, Proceedings of Intemational Joint Conference on Neural Networks, Montreal, Canada, July 31 August 4, 2005.

[6] H.J. Wang, J. Li, H.G. Liu, J.S. Jiang, The Application of FDTD and Micro Genetic Algorithms to the Planar Spiral Inductors, ENGINEERING SCIENCE, 16(11): 13-18, 2004.

[7] T.S. Horng, J.K. Jau, C.H. Huang, and F.Y. Han, Synthesis of a Super Broadband Model for OnChip Spiral Inductors, 2004 IEEE Radio Frequency Integrated Circuits Symposium, TUlD-3

[8] A. Nieuwoudt, Yehia Massoud, Robust Automated Synthesis Methodology for Integrated Spiral Inductors with Variability, 2005. ICCAD-2005. IEEE/ACM International
Conference on Computer-Aided Design, pp. 502 - 507, 2005.

[9] José Ernesto, Rayas-Sánchez, EM-Based Optimization of Microwave Circuits Using Artificial Neural Networks: The State-of-the-Art, IEEE TRANSACTIONS ON MICROWAVE THEORY AND TECHNIQUES, 52(1), JANUARY 2004.

[10] C. Yildiz, Mustafa Turkmen, Very accurate and simple CAD models based on neural networks for coplanar waveguide synthesis, 2005 Wiley Periodicals, Inc. Int $J$ RF and Microwave CAE 15: 218-224, 2005

[11] W. Gao, Scalable Compact Circuit Model for Spiral Inductor-Like Components in CMOS RF ICs , Dissertation Submitted to Tsinghua University in partial fulfillment of the requirement for the degree of Doctor of Engineering, 2006.

[12] M. Lin, Y.M. Li, H.Y. Chen, An Optimization Technique for Planar Spiral Inductor Based on the Inductor's Physical Model and Genetic Algorithm, Chinese Journal of Semiconductors, 22(7):897 903, 2001.

[13] R.J. Pratap, S. Sarkar, Stephane Pinel, Modeling and Optimization of Multilayer RF Passives Using Coupled Neural Networks and Genetic Algorithms, 2004 IEEE MIT-S Digest, 0-78038331-1/04.

[14] S.K. Mandal, A. De, A 20GHZ COMPACT SCALABLE MODEL OF SILICON-BASED ON-CHIP SPIRAL INDUCTOR FOR RFICS, 2005 15th Int. Crimean Conference: Microwave \& Telecommunication Technology (CriMiCo'2005). 12-16 September, Sevastopol, Crimea, Ukraine.

[15] D. Goldberg, Genetic Algorithms in Search, Optimization \& Machine Learning, Mass: Addison Wesley, 1989.

[16] R.J. Pratap, S. Sarkar and S. Pinel, Modeling and optimization of multilayer RF passives using coupled neural networks and genetic algorithms, IEEE MTT-S Digest, 2004. 